\title{
Fluctuations studies in PHOBOS
}

\section{Constantin Loizides ${ }^{4}$ for the PHOBOS collaboration}

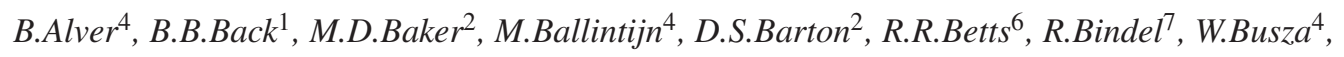
Z.Chai ${ }^{2}$, V.Chetluru ${ }^{6}$, E.García ${ }^{6}$, T.Gburek ${ }^{3}$, K.Gulbrandsen $^{4}$, J.Hamblen $^{8}$, I.Harnarine ${ }^{6}$, C.Henderson ${ }^{4}$, D.J.Hofman ${ }^{6}$, R.S.Hollis ${ }^{6}$, R.Hotyński ${ }^{3}$, B.Holzman ${ }^{2}$, A.Iordanova ${ }^{6}$, J.L.Kane ${ }^{4}$, P.Kulinich ${ }^{4}$, C.M.Kuo ${ }^{5}$, W.Li ${ }^{4}$, W.T.Lin ${ }^{5}$, C.Loizides ${ }^{4}$, S.Manly ${ }^{8}$, A.C.Mignerey ${ }^{7}$, R.Nouicer ${ }^{2}$, A.Olszewski ${ }^{3}$, R.Pak ${ }^{2}$, C.Reed ${ }^{4}$, E.Richardson ${ }^{7}$, C.Roland $^{4}$, G.Roland $^{4}$, J.Sagerer $^{6}$, I.Sedykh $^{2}$, C.E.Smith ${ }^{6}$, M.A.Stankiewicz ${ }^{2}$, P.Steinberg ${ }^{2}$, G.S.F.Stephans ${ }^{4}$, A.Sukhanov ${ }^{2}$, A.Szostak ${ }^{2}$, M.B.Tonjes ${ }^{7}$, A.Trzupek ${ }^{3}$, G.J.van Nieuwenhuizen ${ }^{4}$, S.S.Vaurynovich ${ }^{4}$, R.Verdier ${ }^{4}$, G.I.Veres ${ }^{4}$, P.Walters ${ }^{8}$, E.Wenger ${ }^{4}$, D.Willhelm ${ }^{7}$, F.L.H.Wolfs ${ }^{8}$, B.Wosiek ${ }^{3}$, K.Woźniak ${ }^{3}$, S.Wyngaardt ${ }^{2}$, B. Wystouch ${ }^{4}$

${ }^{1}$ Argonne National Laboratory, Argonne, IL 60439-4843, USA

2 Brookhaven National Laboratory, Upton, NY 11973-5000, USA

${ }^{3}$ Institute of Nuclear Physics PAN, Kraków, Poland

${ }^{4}$ Massachusetts Institute of Technology, Cambridge, MA 02139-4307, USA

${ }^{5}$ National Central University, Chung-Li, Taiwan

${ }^{6}$ University of Illinois at Chicago, Chicago, IL 60607-7059, USA

${ }^{7}$ University of Maryland, College Park, MD 20742, USA

${ }^{8}$ University of Rochester, Rochester, NY 14627, USA

We will briefly report on various correlation and fluctuations analyses that currently are being developed, or recently have been published, by the PHOBOS experiment on $200 \mathrm{GeV} \mathrm{Au+Au}$ collisions at RHIC. In particular, we will discuss our search for rare events, forward-backward multiplicity and two-particle angular correlations, as well as our studies of eccentricity and elliptic flow fluctuations.

Correlations and Fluctuations in Relativistic Nuclear Collisions

July 7-9 2006

Florence, Italy 


\section{Introduction}

The research of heavy-ion collisions at ultra-relativistic energies combines experimental and theoretical efforts in the understanding of the phase-space properties of strongly-interacting matter. At extreme conditions of high temperature and density (relative to normal nuclear matter), lattice QCD calculations predict a phase transition to a system dominated by partonic, rather than hadronic, degrees of freedom. Indeed, the most important conclusion from the experimental programme at the Relativistic Heavy Ion Collider (RHIC) is [1] that - at least in central $\mathrm{Au}+\mathrm{Au}$ collisions at $200 \mathrm{GeV}$ collision energy - an extremely dense, highly interacting system is created, reaching energy densities much larger than $\sim 1 \mathrm{GeV} / \mathrm{fm}^{3}$, the characteristic scale for the QCD phase transition.

Most PHOBOS studies that have contributed to the understanding of the properties of the created matter are dealing with the measurement and analysis of single-particle distributions, averaged over many events. In particular, many of our physics conclusions are based on scaling observations that are directly linked to the data. The observed scaling rules reveal common features in heavy-ion collisions $(\mathrm{Cu}+\mathrm{Cu}, \mathrm{Au}+\mathrm{Au})$ and allow for the comparison with simpler systems $(\mathrm{d}+\mathrm{Au}, \mathrm{p}+\mathrm{p})$ in a broad range of collision energies $\left(\sqrt{s_{\mathrm{NN}}}=19.6\right.$ to $\left.200 \mathrm{GeV}\right)$. For a recent summary, see Ref. [1, 2] and references therein.

In these conference proceedings, we will briefly report on various PHOBOS analyzes that extract information from the recorded data using correlation techniques involving the measurement of event-by-event, rather than event-averaged quantities. In particular, we will focus on studies of correlations and fluctuations that contain a wealth of detailed information concerning the underlying mechanism of particle production in collisions of both, heavy ions and simpler systems. The uniquely broad pseudo-rapidity $(\eta)$ coverage of the PHOBOS multiplicity detector $(|\eta| \leq 5.4)$ enables a wide variety of such studies. It consists of single-layer Silicon-pad detectors supported in an octagonal frame surrounding the interaction region as well as in ring-shaped frames arranged along the beam pipe, described in detail in Ref. [3].

\section{Unusual event search}

Since the average properties of the pseudo-rapidity and multiplicity distributions are known with good precision [1,2], we can perform studies of unusual properties in these distributions with respect to the average. As reported in Ref. [4], our first investigation aims for events with unusually high total number of charged particles. Using the data for the most central 3\% Au+Au collisions from the high statistics run at $\sqrt{s_{N N}}=200 \mathrm{GeV}$, a total of about 2 million events, we observe at the end of steeply falling multiplicity distribution a clear, but small, tail of events, which seem to deviate from the general trend, extending to high multiplicity. As a preliminary conclusion, we find that these events are rarer than a few times $10^{-4}$. One obvious source of such events is pile-up in which two collisions occur within the read-out time of the experiment.

An additional study searches for events with unusual shapes of the $\mathrm{dN} / \mathrm{d} \eta$ distribution. Events from the same central $\mathrm{Au}+\mathrm{Au}$ sample are used. Within bins in vertex location (to eliminate trivial acceptance effects), the average $\mathrm{dN} / \mathrm{d} \eta$-shape and variance are determined. After normalizing each

event to the same total number of hits, we calculate the $\chi^{2}$ to compare to the average event shape. A 
clear excess of events with large $\chi^{2}$ is observed, about 200 in total, which translates into a slightly smaller preliminary limit of about $1 \times 10^{-4}$. However, as with the high multiplicity events, the number of these unusual events is found to be correlated with the event rate. The extrapolation of the number of these events to low collisions rate is close to zero, which suggests the possibility of smaller values.

\section{Forward-backward correlations}

Charged-particle production is highly correlated over large regions of rapidity. Thus, the study of forward-backward multiplicity correlations addresses the underlying structure of the singleparticle distribution. We perform an event-by-event comparison of the integrated multiplicity $N_{F}$, in a bin defined in the forward $(\eta>0)$ region, centered at $\eta$ with pseudo-rapidity interval $\Delta \eta$, with the multiplicity $N_{B}$, measured in an identical bin defined in the backward hemisphere, centered at negative $\eta$. With these definitions, we construct the event-wise observable $C=\left(N_{F}-N_{B}\right) / \sqrt{N_{F}+N_{B}}$, and measure the variance $\sigma_{C}^{2}$ for a set of events with nominally similar characteristics (e.g. collision centrality).

The $C$ variable is sensitive to various types of long and short range correlations. An "intrinsic" long-range correlation in the emission of particles into the forward and backward hemisphere from a single source would give $N_{F}-N_{B}=0$ with a substantial value of $N_{F}+N_{B}$, thus forcing $C$ to 0 . However, if the particle sources would produce particles into the forward or backward region, such that the partitioning was binomial, this would lead to $\sigma_{C}^{2}=1$, since $\sigma^{2}\left(N_{F}-N_{B}\right)=N_{F}+N_{B}$ in that case. Short-range correlations would arise if objects emitted into either hemisphere break into $\langle k\rangle$ particles on average, with a variance of $\sigma_{k}^{2}$, each of which remains close in rapidity (e.g. due to isotropic emission). Such decay of "clusters" has a particular effect on $\sigma_{C}^{2}$. Consider the idealized case, where clusters are independently produced and subsequently decay into exactly $k$ particles, with all of the $N$ particles distributed into the same $\eta$-bins in the forward or backward direction. In this case, the underlying fluctuations are those associated with $N / k$ objects rather than $N$ independent particles, and $C \rightarrow \sqrt{k} C$. It follows, that $\sigma_{C}^{2}$ is linear with the cluster multiplicity. In a more realistic physical situation, particles from each cluster may fall outside the chosen $\eta$-bin, or even in the opposite hemisphere. This results in a non-trivial modification of the measured value of $k$, but one which is still related directly to the cluster multiplicity, or rather to the effective cluster size $\left(k_{\text {eff }}\right)$. The effective cluster size incorporates the effect of the multiplicity per cluster having its own distribution.

The results of our measurement are published in Ref. [5] using the $200 \mathrm{GeV} \mathrm{Au+Au} \mathrm{data}$ taken during Runs 2 and 4, in 2001 and 2004, respectively. The $\sigma_{C}^{2}$ values extracted as a function of both $\eta$ and $\Delta \eta$, for two centrality bins, are corrected for all detector effects and, thus, can be directly compared to phenomenological models. We observe significant short-range correlations at all centralities and pseudo-rapidities, rather than just at mid-rapidity, where those short-range correlations are trivially expected. There is a non-trivial centrality and rapidity dependence of these correlations, in both $\eta$ and $\Delta \eta$. We find that neither HIJING nor AMPT reproduces all of the main qualitative features, but the way in which they fail to do so may well provide information on the underlying physics. It is expected that QGP formation would modify the measured properties 

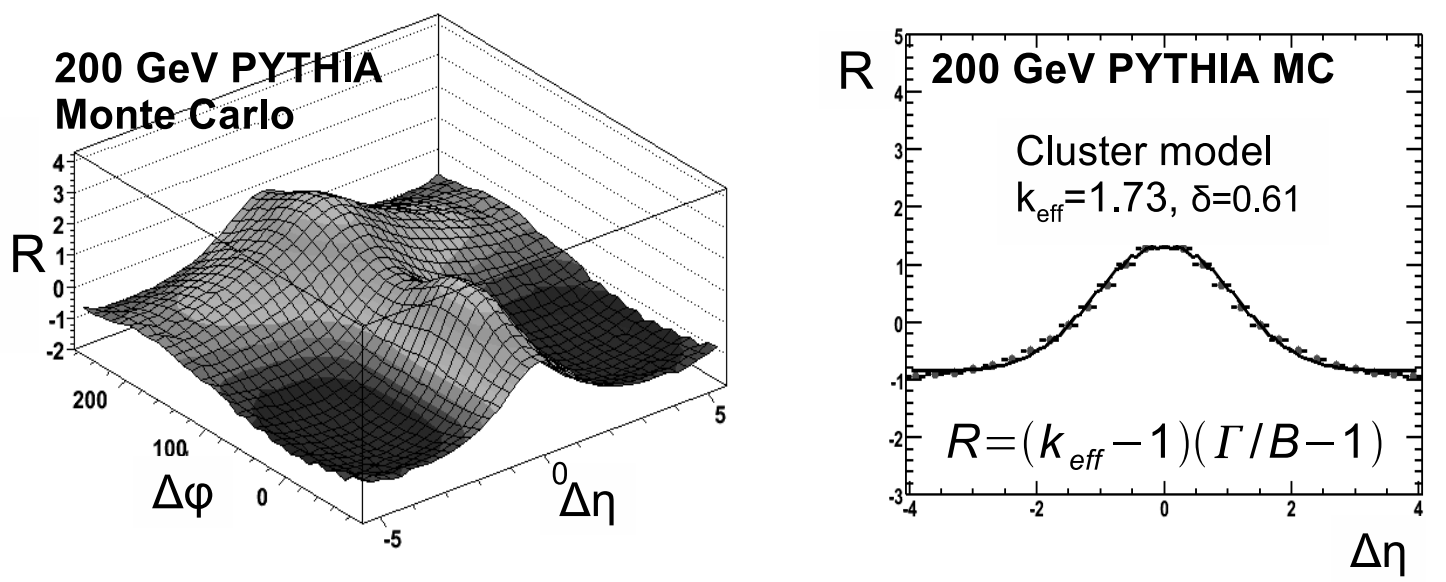

Figure 1: (Left) Two-particle correlation function as a function of $\Delta \phi$ and $\Delta \eta$ for $200 \mathrm{GeV}$ pp Monte Carlo events (on particle level). (Right) The projection of the two-particle correlation function onto $\Delta \eta$ together with a fit to the independent cluster model.

of clusters [6]. Our data provides means to study such effects, and — to the very least— set upper limits on their occurrence.

\section{Two-particle correlations}

As first reported in these proceedings [7], we recently have made efforts to extend our studies from correlations in pseudo-rapidity to the two-dimensional measurement of two-particle angular correlation functions.

For these studies, we define the two-dimensional correlation function $R=\left\langle(n-1) F_{n} / B-1\right\rangle$, in $\Delta \phi$ and $\Delta \eta$, where $F_{n}(B)$ is the foreground (background) correlation function (see Fig. 1, left). The foreground distribution is determined by correlating particle pairs from the same event, while the background distribution is determined by particle pairs formed from mixed events. To compensate for the dilution of the correlation function due to the rapid increase of uncorrelated pairs with increasing multiplicity $(n)$, we use the multiplicity-weighted average in the definition of $R$.

The advantage of PHOBOS for performing such a measurement clearly is the large pseudorapidity coverage that allows us to study particle production over large ranges in $\Delta \eta$. However, since we only have a single hit in the silicon layer instead of a well defined track, special care is needed for the treatment of secondaries that pollute the correlation signal.

Currently, we measure the correlation function without the usage of a trigger particle to be sensitive to soft physics processes. To be quantitative, we study the projection of $R$ onto $\Delta \eta$, and relate its properties to an independent cluster emission model. In the context of this model, $R=\left(k_{\text {eff }}-1\right)(\Gamma / B-1)$, where $\Gamma \propto \exp \left(-\Delta \eta^{2} /(2 \delta)^{2}\right)$ characterizes the correlation of particles produced by a single cluster with a decay width of $2 \delta$ and an effective cluster multiplicity, $k_{\text {eff }}$. An example of such a fit is shown in Fig. 1 on the right for $200 \mathrm{GeV}$ pp Monte Carlo events. 


\section{Eccentricity fluctuations}

PHOBOS has measured elliptic flow as a function of pseudo-rapidity, centrality, transverse momentum, center-of-mass energy [8,9,10] and, recently, nuclear species [11]. In particular, the measurements of elliptic flow as a function of centrality provide information on how the azimuthal anisotropy of the initial collision region drives the azimuthal anisotropy in particle production.

The azimuthal anisotropy of the initial collision region can be characterized by the eccentricity $(\varepsilon)$ of the overlap region of the colliding nuclei in the transverse plane. The strength of the elliptic flow, $v_{2}$, is commonly defined by the coefficient of the second harmonic in the Fourier expansion of the azimuthal angle distribution relative to the reaction plane, $\Psi_{\mathrm{R}}$, such that $v_{2}=\left\langle\cos \left(2 \phi-2 \Psi_{\mathrm{R}}\right)\right\rangle[12]$. From hydrodynamical calculations [13] we expect that $v_{2}$ scales approximately with $\varepsilon$ for small $\varepsilon$. Such calculations typically use smooth, event-averaged initial conditions, for which the initial azimuthal asymmetry is well described by the "standard" eccentricity,

$$
\varepsilon_{\mathrm{std}}=\frac{\sigma_{y}^{2}-\sigma_{x}^{2}}{\sigma_{x}^{2}+\sigma_{y}^{2}},
$$

where $\sigma_{x}^{2}\left(\sigma_{y}^{2}\right)$ is the variance of the participant nucleon distributions projected on the $x(y)$ axis, taken to be along (perpendicular to) the impact parameter direction.

In Fig. 2a, we show the centrality dependence of $v_{2}$ at mid-rapidity $(|\eta|<1)$ for $\mathrm{Cu}+\mathrm{Cu}$ and $\mathrm{Au}+\mathrm{Au}$ at $\sqrt{s_{\mathrm{NN}}}=62.4$ and $200 \mathrm{GeV}$ collision energies, as obtained from our hit-based analysis method $[9,11]$. A substantial flow signal is measured in $\mathrm{Cu}+\mathrm{Cu}$ at both energies, even for the most central events. This is quite surprising, as according to the initial anisotropy given by $\varepsilon_{\text {std }}$ one expects that $v_{2}$ should approach zero as the collisions become more central, as it is found in the $\mathrm{Au}+\mathrm{Au}$ data.

As a possible explanation for the large $v_{2}$ signal in the small $\mathrm{Cu}+\mathrm{Cu}$ system, we have argued that event-by-event fluctuations in the shape of the initial collision region may drive the elliptic flow [11, 14]. For small systems or small transverse overlap regions, fluctuations in the nucleon positions frequently create a situation where the minor axis of the overlap ellipse is not aligned with the impact parameter vector. These fluctuations are neglected in the definition of $\varepsilon_{\text {std. }}$. To study initial-geometry fluctation effects, we have introduced the "participant eccentricity",

$$
\varepsilon_{\mathrm{part}}=\frac{\sqrt{\left(\sigma_{y}^{2}-\sigma_{x}^{2}\right)^{2}+4 \sigma_{x y}^{2}}}{\sigma_{x}^{2}+\sigma_{y}^{2}} .
$$

This definition accounts for the nucleon position fluctuations by quantifying the eccentricity eventby-event with respect to the minor axis of the overlap ellipse, in the frame, defined by $\Psi_{\text {part }}$, that diagonalizes the ellipse. Note, $\sigma_{x y}=\langle x y\rangle-\langle x\rangle\langle y\rangle, \sigma_{x}^{2}$ and $\sigma_{y}^{2}$ are the (co-)variances of the $x$ and $y$ participant nucleon position distributions expressed in the original frame, given by $\Psi_{\mathrm{R}}$. For a system with a large number of nucleons, the covariance term is comparatively small. Therefore, the average values of $\varepsilon_{\text {std }}$ and $\varepsilon_{\text {part }}$ over many events are similar for all, but the most peripheral interactions for the $\mathrm{Au}+\mathrm{Au}$ system. For the smaller $\mathrm{Cu}+\mathrm{Cu}$ system, however, fluctuations in the nucleon positions are important for all centralities [11].

In Fig. 2b, we show a Glauber model calculation of $\varepsilon_{\text {part }}$ as a function of $N_{\text {part }}$ for $\mathrm{Cu}+\mathrm{Cu}$ and $\mathrm{Au}+\mathrm{Au}$. The colliding nuclei are built by randomly placing nucleons according to a Woods-Saxon 


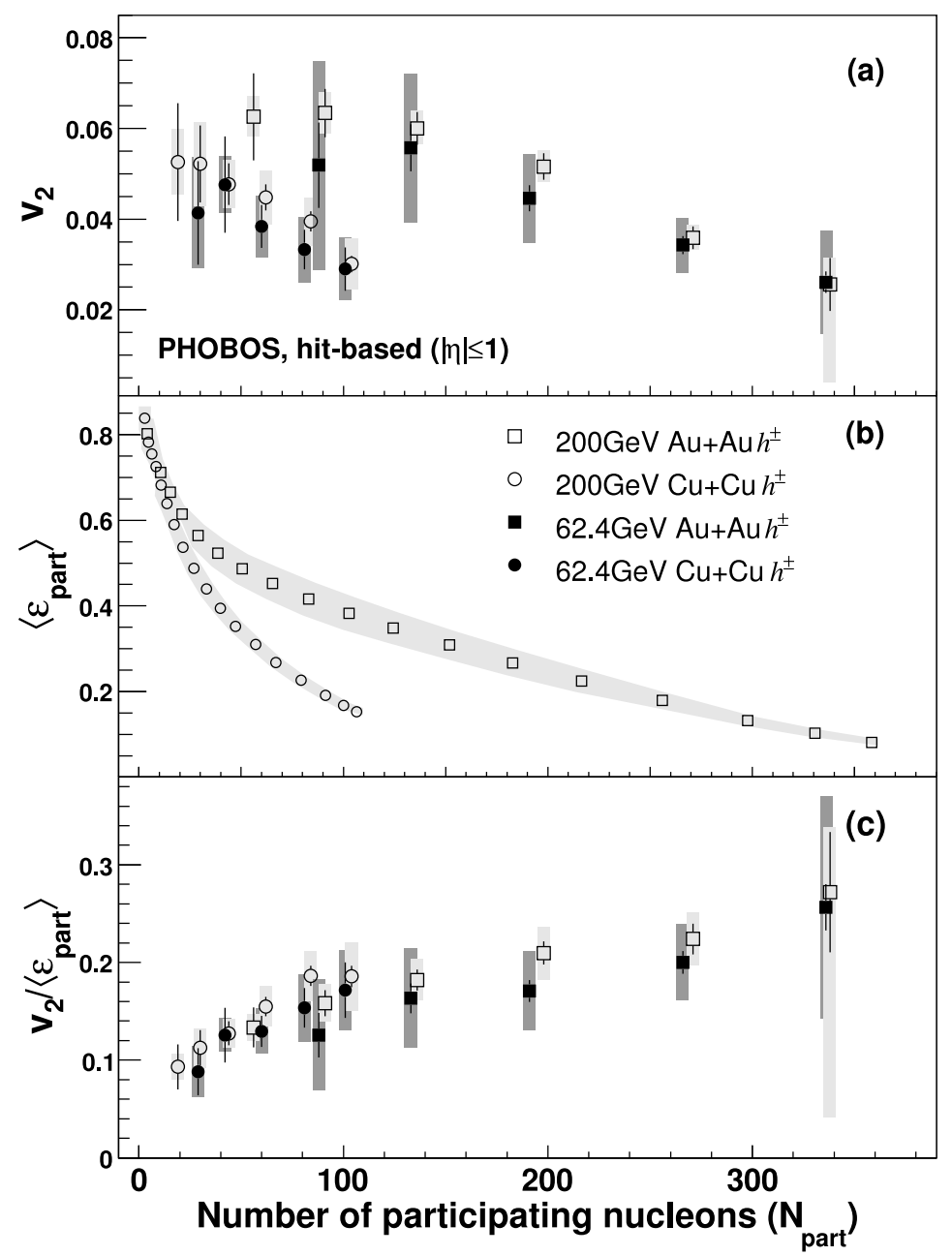

Figure 2: (a) $v_{2}$ (unscaled), (b) $\left\langle\varepsilon_{\text {part }}\right\rangle$ and (c) $v_{2} /\left\langle\varepsilon_{\text {part }}\right\rangle$ vs. $\mathrm{N}_{\text {part }}$ for $\mathrm{Cu}+\mathrm{Cu}$ and $\mathrm{Au}+\mathrm{Au}$ collisions at $\sqrt{s_{\mathrm{NN}}}=$ 62.4 and $200 \mathrm{GeV}$. 1- $\sigma$ statistical errors (bars) and 90\% C.L. systematic errors (bands) are shown. Data points and eccentricity calculation are from Refs. [9, 11].

distribution. Excluded volume effects are addressed by requiring a minimum inter-nucleon separation distance of $0.4 \mathrm{fm}$. As opposed to $\varepsilon_{\text {std }}$, where averages are implicitly over participants and events, the variance expressions in $\varepsilon_{\text {part }}$ are averaged event-by-event over participants, individually. To check how the event-by-event interpretation of the Glauber calculation depends on the external parameter settings, we varied a number of sources of systematic error, like the nuclear radius, nuclear skin depth, nucleon-nucleon inelastic cross-section $\sigma_{\mathrm{NN}}$ and minimum nucleon separation. Varying each specific parameter within reasonable limits, the individual contributions were added in quadrature to determine the $90 \%$ confidence level errors shown in Fig. 2 b.

In order to compare the elliptic flow signals across nuclear species and with hydrodynamical predictions, it is important to scale out the difference in the initial asymmetry of the collision geometry. In Fig. $2 c$, we show the eccentricity-scaled flow for $\mathrm{Cu}+\mathrm{Cu}$ and $\mathrm{Au}+\mathrm{Au}, v_{2} /\left\langle\varepsilon_{\text {part }}\right\rangle$, as a function of centrality. The scaled data are very similar for both the $\mathrm{Cu}+\mathrm{Cu}$ and $\mathrm{Au}+\mathrm{Au}$ collision systems at the same number of participants. It is important to note that the apparent scaling does 

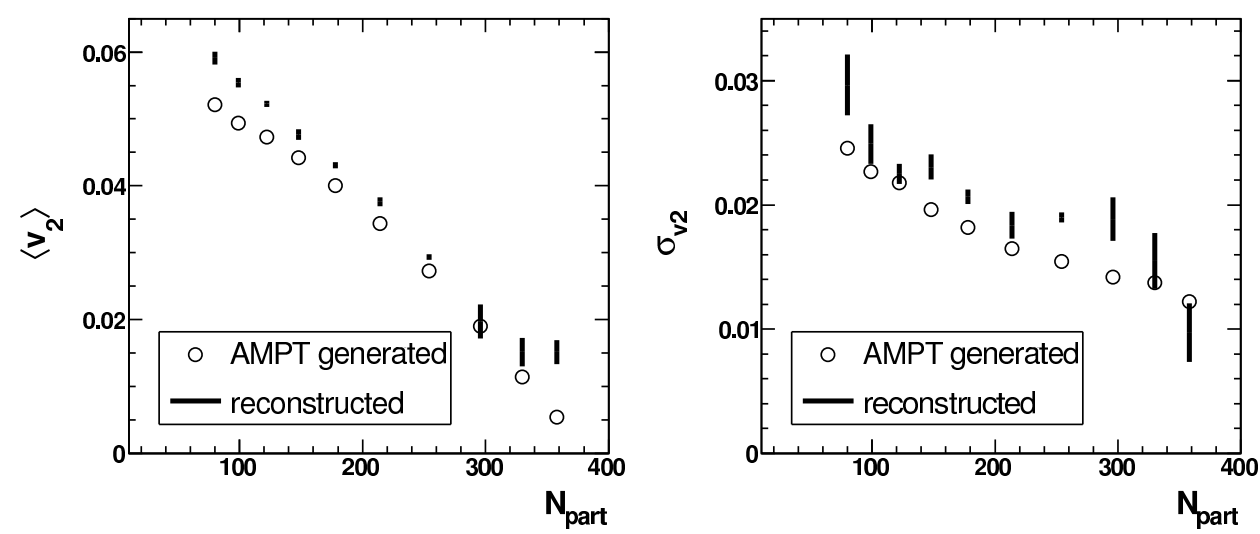

Figure 3: Generated and reconstructed $\left\langle v_{2}\right\rangle$ (left) and $\sigma_{v_{2}}$ for AMPT vs. $N_{\text {part }}$. The generated signal is extracted from a mixed-event analysis based on the true Monte Carlo particle information. The reconstructed signal is averaged over results of running the complete flow fluctuation analysis in 10 vertex bins using the kernel created from the modified HIJING samples. The black lines represent the $\pm 1 \sigma$ error on the mean, with the mean centered (not shown).

not rely on the fine-tuning of the Glauber parameter settings, as is evident from the systematic errors, which are rather small (see Fig. 2b).

\section{Elliptic flow fluctuations}

Our $\left\langle v_{2}\right\rangle$ analysis suggests that the proposed participant fluctuations are driving the value of $v_{2}$ event-by-event, thus we expect them to contribute to event-by-event elliptic flow fluctuations. As mentioned above, ideal hydrodynamics leads to $v_{2} \propto \varepsilon$ [13]. Assuming the same relation holds event-by-event, this would imply that $\sigma_{v_{2}} /\left\langle v_{2}\right\rangle=\sigma_{\varepsilon} /\langle\varepsilon\rangle$, where $\sigma_{v_{2}}\left(\sigma_{\varepsilon}\right)$ is the standard deviation of the event-by-event distribution of $v_{2}(\varepsilon)$. Using our $\left\langle v_{2}\right\rangle$ data (Fig. 2a) and the Monte Carlo Glauber simulation to obtain $\sigma_{\varepsilon}$ (and $\langle\varepsilon\rangle$ ) for the participant eccentricity, we estimate $\sigma_{v_{2}}$ to be about $2 \%$ for all centralities, except the most central Au+Au collisions at $\sqrt{s_{\mathrm{NN}}}=200 \mathrm{GeV}$. This estimate leads to rather large relative fluctuations $\left(\sigma_{v_{2}} /\left\langle v_{2}\right\rangle\right)$ between 35 and $50 \%$. These estimates neglect other sources of elliptic flow fluctuations. The measurement of $v_{2}$ fluctuations as a probe of early stage dynamics of heavy-ion collisions has been suggested earlier by Mrowczynski and Shuryak [15], although neglecting the possibility for eccentricity fluctuations.

In the following, we will give a brief overview of the analysis method that we have developed to measure flow fluctuations with the PHOBOS detector in peripheral and semi-central $\mathrm{Au}+\mathrm{Au}$ collisions. Details can be found in Ref. [16].

We seek to discriminate known (statistical) from unknown (presumably dynamical) contributions to observed flow fluctuations. Ideally, they would add according to $\sigma_{v_{2}, \text { obs }}^{2}=\sigma_{v_{2} \text {,dyn }}^{2}+\sigma_{v_{2} \text {,stat }}^{2}$. This relation holds if the average of the measurement, $\left\langle v_{2}^{\mathrm{obs}}\right\rangle$, gives the true average in the data, $\left\langle v_{2}\right\rangle$, and if the resolution of our method is independent of the true value. Neither of these conditions are fully satisfied in the event-by-event measurement of $v_{2}$. Therefore, a more detailed knowledge of the response function is required. We define $K\left(v_{2}^{\text {obs }}, v_{2}, n\right)$ as the distribution of the event-by-event 
observed elliptic flow, $v_{2}^{\text {obs }}$, for events with constant input flow value, $v_{2}$, and multiplicity, $n$. Assuming a set of events has an input $v_{2}$ distribution given by $f\left(v_{2}\right)$, then $g\left(v_{2}^{\text {obs }}\right)$, the distribution of $v_{2}^{\text {obs }}$, will be given according to

$$
g\left(v_{2}^{o b s}\right)=\int_{0}^{\infty} K\left(v_{2}^{\text {obs }}, v_{2}, n\right) f\left(v_{2}\right) N(n) \mathrm{d} v_{2} \mathrm{~d} n,
$$

where $N(n)$ is the multiplicity distribution of the events in the given set of events (centrality bin). Thus, our event-by-event elliptic flow fluctuation analysis consists of three steps:

- Finding the observed $v_{2}$ distribution, $g\left(v_{2}^{\mathrm{obs}}\right)$, for a set of events by an event-by-event measurement of $v_{2}^{\text {obs }}$.

- Construction of the kernel, $K\left(v_{2}^{\mathrm{obs}}, v_{2}, n\right)$, by studying the detector response for sets of constant (known) input value of $v_{2}$ and multiplicity $n$.

- Calculating the true $v_{2}$ distribution, $f\left(v_{2}\right)$, by finding a solution to eq. (6.1).

For the event-by-event measurement we use a maximum likelihood method, making use of all hit information from the multiplicity array to measure a single value, $v_{2}^{o b s}$, while allowing an efficient correction for the non-uniformities in the acceptance. We model the measured pseudorapidity dependence of $v_{2}$ according to $v_{2}(\eta)=v_{2} \cdot(1-|\eta| / 6)$, with $v_{2} \equiv v_{2}(0)$. We define the probability distribution function (PDF) of a particle to be emitted in the direction $(\eta, \phi)$ for an event with $v_{2}$ and reaction plane angle $\phi_{0}$ as $P\left(\eta, \phi \mid v_{2}, \phi_{0}\right)=p(\eta)\left[1+2 v_{2}(\eta) \cos \left(2 \phi-2 \phi_{0}\right)\right]$. The normalization $p(\eta)$ is constructed such that the PDF, folded with the PHOBOS acceptance, yields the same value for different sets of parameters $\left(v_{2}, \phi_{0}\right)$. Maximizing $\prod_{i=1}^{n} P\left(\eta_{i}, \phi_{i} \mid v_{2}, \phi_{0}\right)$ as a function of $v_{2}$ and $\phi_{0}$ allows us to measure $v_{2}^{\text {obs }}$ event-by-event.

We determine the response function $K\left(v_{2}^{\text {obs }}, v_{2}, n\right)$ in bins of $v_{2}$ and $n$ using modified HIJING events. Flow of constant magnitude $\left(v_{2}\right)$ with a flat reaction plane distribution $\left(\phi_{0}\right)$ is introduced into generated HIJING $\mathrm{Au}+\mathrm{Au}$ events. This is achieved by redistributing the resulting particles in each event in $\phi$ randomly according to $1+2 v_{2}(\eta) \cos \left(2 \phi-2 \phi_{0}\right)$, using their generated $\eta$ positions. The modified HIJING events are run through GEANT to obtain the PHOBOS detector response.

To finally extract $f\left(v_{2}\right)$, we assume a Gaussian distribution, with two parameters, $\left\langle v_{2}\right\rangle$ and $\sigma_{v_{2}}$. For given values of $\left\langle v_{2}\right\rangle$ and $\sigma_{v_{2}}$, it is possible to take the integral in eq. (6.1) to obtain the expected distribution, $g_{\exp }\left(v_{2}^{\mathrm{obs}} \mid\left\langle v_{2}\right\rangle, \sigma_{v_{2}}\right)$. Comparing the expected and observed distributions, the values for $\left\langle v_{2}\right\rangle$ and $\sigma_{v_{2}}$ are obtained from a minimized $\chi^{2}$ of the data to the expectation.

As outlined in Ref. [16], the whole analysis procedure was verified on similar HIJING events as used to construct the kernel, and — for that rather ideal situation - found to be successfully reconstructing the input fluctuations, provided $\left\langle v_{2}\right\rangle \geq 0.03$. Here, we report on a different study where we have used fully simulated AMPT events to verify the complete analysis chain with a different set (and type) of "data" events than used to create the kernel. The analysis is done in 10 bins of collision vertex of $2 \mathrm{~cm}$. Fig. 3 shows the averaged results obtained from the different vertex bins compared to the generated signal. Since the information about the generated $v_{2}$ in AMPT has not been readily available on an event-by-event basis, we extracted the generated $\left\langle v_{2}\right\rangle$ and $\sigma_{v_{2}}$ from a mixed-event analysis based on the true Monte Carlo particle information. We conclude that the developed analysis chain is able to reconstruct the fluctuations to a satisfactory degree over a large 
range in centrality, also in samples of "data" that are different from the ones used to construct the kernel.

\section{Summary}

PHOBOS has recently developed a rich programme of correlation studies that address different aspects of particle production in elementary and heavy-ion collisions. Our studies of forwardbackward multiplicity and two-particle pseudo-rapidity correlations deal with the underlying structure of the single-particle distribution and will allow us to compare particle production mechanism over large ranges in pseudo-rapidity in various systems.

As argued earlier, fluctuations in the initial collision geometry can explain the large observed elliptic flow in $\mathrm{Cu}+\mathrm{Cu}$ relative to $\mathrm{Au}+\mathrm{Au}$ collisions, especially for most central collisions. These fluctuations in the inital state may be the dominant source for rather large elliptic flow fluctuations. We have recently developed a complete analysis chain for the measurement of dynamical elliptic flow fluctuations that is ready to be used in the analysis of PHOBOS data.

\section{References}

[1] B. Back et al. [PHOBOS], Nucl. Phys. A 757, 28 (2005).

[2] G. Roland et al. [PHOBOS], Nucl. Phys. A 774, 113 (2006) [arXiv:nucl-ex/0510042].

[3] B. B. Back et al. [PHOBOS], Nucl. Instrum. Methods A 499, 603 (2003).

[4] G. S. F. Stephans et al. [PHOBOS], Nucl. Phys. A 774, 639 (2006).

[5] B. B. Back et al. [PHOBOS], Phys. Rev. C 74, 011901 (2006).

[6] L. J. Shi and S. Jeon, Phys. Rev. C 72, 034904 (2005).

[7] W. Li [PHOBOS], these proceedings.

[8] B. B. Back et al. [PHOBOS], Phys. Rev. Lett. 89, 222301 (2002).

[9] B. B. Back et al. [PHOBOS], Phys. Rev. Lett. 95, 122303 (2005).

[10] B.B. Back et al. [PHOBOS], Phys. Rev. C 72, 051901(R) (2005).

[11] B. Alver et al. [PHOBOS], submitted to Phys. Rev. Lett., arXiv:nucl-ex/0610037.

[12] A.M. Poskanzer and S.A. Voloshin, Phys. Rev. C 58, 1671 (1998).

[13] R. S. Bhalerao et al., Phys. Lett. B 627, 49 (2005).

[14] S. Manly et al. [PHOBOS], Nucl. Phys. A 774, 523 (2006).

[15] S. Mrowczynski and E. Shuryak, Acta Phys.Polon. B34 4241 (2003).

[16] B. Alver et al. [PHOBOS], these proceedings, arXiv:nucl-ex/0608025. 